\title{
PERIODICITY PROBLEM OF SUBSTITUTIONS OVER TERNARY ALPHABETS*
}

\author{
BO TAN $^{1}$ AND ZHI-YING WeN $^{2}$
}

\begin{abstract}
In this paper, we characterize the substitutions over a three-letter alphabet which generate a ultimately periodic sequence.
\end{abstract}

Mathematics Subject Classification. 11B85, 68R15.

The study of periodicity is a central topic in combinatorics and presents some important applications in algebra, in formal language theory and in string searching algorithms. In this paper, we study the substitutions which generate a periodic sequence.

\section{INTRODUCTION}

Let $S$ be the alphabet of finite letters. Let $S^{*}$ and $\tilde{S}$ be respectively the free monoid and the free group generated by $S$. The empty word $\varepsilon$ is their neutral element. Let $S^{+}=S^{*} \backslash\{\varepsilon\}$. Let $S^{\mathbb{N}}$ be the set of infinite sequences on $S$.

Let $v$ and $w$ be two words. We say that $v$ is a factor of $w$ and then write $v \prec w$, if there exist $u, u^{\prime} \in S^{*}$, such that $w=u v u^{\prime}$. We say that $v$ is a prefix (resp. suffix) of a word $w$ and then note $v \triangleleft w$ (resp. $v \triangleright w$ ), if there exists $u \in S^{*}$ such that $w=v u$ (resp. $w=u v$ ). We say that $v$ is a proper factor of $w$ if $v \prec w$ and $v \neq w$. Likewise, we define the notions of proper prefix and proper suffix. The notions of prefix and factor extend in a natural way to sequences.

A morphism $\varphi: S^{*} \rightarrow S^{*}$ is called a substitution of $S^{*}$. In this work, except in the last section, we deal only with non-erasing substitutions, which means that the image of any letter is different from the empty word $\varepsilon$.

Keywords and phrases. Periodicity, substitution.

* Supported by NSFC No. 10501035, 10571104 and 10631040.

1 Department of Mathematics, Huazhong University of Science and Technology, Wuhan 430074, P.R. China; bo_tan@163.com

2 Department of Mathematical Sciences, Tsinghua University, Beijing 100084, P.R. China; wenzy@tsinghua.edu.cn 
Let $\varphi$ be a substitution. If there is a letter $a \in S$ such that the word $\varphi(a)$ begins with $a$ and is of length at least equal to 2 , in other words,

$$
\varphi(a)=a v \text { for some } v \in S^{+}
$$

then, for $n \geq 1, \varphi^{n}(a)$ is a prefix of $\varphi^{n+1}(a)$, and thus there is a unique (infinite) sequence $\xi$ beginning with $a$ such that $\varphi(\xi)=\xi$. In this case, we say that the substitution $\varphi$ generates, with the axiom $a$, the sequence $\xi$. And we write $\xi=\varphi^{\infty}(a)$.

Ultimately periodic sequences are sequences of the lowest (subword) complexity. In this paper we study the substitutions which generate a ultimately periodic sequence. The decidability result on this kind of problem is proved independently by, on the one hand, Harju and Linna [2] and, on the other hand, Pansiot [6], that is, they give an algorithm which, for a substitution, determines whether this substitution has a ultimately periodic fixed point or not. And Lando [4] provides the bounds for the period and index of the substitution.

Finding all substitutions which generate a ultimately periodic sequence is a quite different problem from determining whether a given substitution generates a ultimately periodic sequence or not.

In the binary alphabet case, Séébold [8] has found all the substitutions which generate a ultimately periodic sequence:

Theorem 0.1. Let $\varphi$ be a (non-erasing) substitution over a two-letter alphabet $\{a, b\}$. Then $\varphi$ generates, with the axiom a, a ultimately periodic sequence $\xi$ if and only if $\varphi$ has one of the following forms:

1. $\varphi(a)=a^{p}, \varphi(b) \in\{a, b\}^{+}, p \geq 2$, and $\xi=a^{\infty}$;

2. $\varphi(a)=a b^{p}, \varphi(b)=b^{q}, p, q \geq 1$, and $\xi=a b^{\infty}$;

3. $\varphi(a)=(a v)^{p}, \varphi(b)=(a v)^{q}, p, q \geq 1, v \in\{a, b\}^{+}$, and $\xi=(a v)^{\infty}$;

4. $\varphi(a)=(a b)^{p} a, \varphi(b)=(b a)^{q} b, p, q \geq 1$, and $\xi=(a b)^{\infty}$;

5. $\varphi(a)=\left(a b^{p}\right)^{q} a, \varphi(b)=b, p, q \geq 1$, and $\xi=\left(a b^{p}\right)^{\infty}$.

In this paper, we consider the same problem over a ternary alphabet and our main result is:

Theorem 0.2. Let $\varphi$ be a (non-erasing) substitution over $S$. Suppose that $\varphi$ generates, with the axiom a, a sequence $\xi$. Then $\xi$ is ultimately periodic if and only if there is a permutation $(x, y)$ of the letters $(b, c)$ such that $\varphi$ has one of the following forms:

1. $\varphi(a)=a^{k}, \varphi(x) \in S^{+}, \varphi(y) \in S^{+}(k \geq 2)$, and $\xi=a^{\infty}$;

2. $\varphi(a)=a x^{k}, \varphi(x) \in x^{+}, \varphi(y) \in S^{+}(k \geq 1)$, and $\xi=a x^{\infty}$;

3. $\varphi(a)=(a v)^{p}, \varphi(x)=(a v)^{q}, \varphi(y) \in S^{+}\left(v \in\{a, x\}^{+}, p, q \geq 1\right)$, and $\xi=(a v)^{\infty}$;

4. $\varphi(a)=(a x)^{p} a, \varphi(x)=(x a)^{q} x, \varphi(y) \in S^{+}(p, q \geq 1)$, and $\xi=(a x)^{\infty}$;

5. $\varphi(a)=\left(a x^{p}\right)^{q} a, \varphi(x)=x, \varphi(y) \in S^{+}(p, q \geq 1)$, and $\xi=\left(a x^{p}\right)^{\infty}$;

6. $\varphi(a)=a v, \varphi(x)=x, \varphi(y)=y\left(v \in\{x, y\}^{+}\right)$, and $\xi=a v^{\infty}$;

7. $\varphi(a)=a v, \varphi(x)=y, \varphi(y)=x\left(v \in\{x, y\}^{+}\right)$, and $\xi=a(v \varphi(v))^{\infty}$;

8. $\varphi(a)=a v, \varphi(x) \in r^{+}, \varphi(y) \in r^{+}\left(v, r \in\{x, y\}^{+}\right)$, and $\xi=a v r^{\infty}$; 
9. $\varphi(a)=a(x y)^{n}, \varphi(x)=(x y)^{l} x, \varphi(y)=(y x)^{m} y(l, n \geq 1, m \geq 0)$, and $\xi=$ $a(x y)^{\infty}$;

10. $\varphi(a)=a(x y)^{n} x, \varphi(x)=y(x y)^{l}, \varphi(y)=x(y x)^{m}(l \geq 1, m, n \geq 0)$, and $\xi=a(x y)^{\infty}$;

11. $\varphi(a)=a\left(y^{i} x y^{l-i}\right)^{n}, \varphi(x)=\left(x y^{l}\right)^{m} x, \varphi(y)=y(l, m, n \geq 1,0 \leq i \leq l)$, and $\xi=a y^{i}\left(x y^{l}\right)^{\infty}$

12. $\varphi$ is ultimately cyclic, in other words, $\varphi^{2}(a) \in w^{+}, \varphi^{2}(x) \in w^{+}, \varphi^{2}(y) \in w^{+}$ $\left(w \in S^{+}, a \triangleleft w\right)$, and $\xi=w^{\infty}$;

13. $\varphi(a)=(a x y)^{l} a, \varphi(x)=(x y a)^{m} x, \varphi(y)=(y a x)^{n} y(l, m, n \geq 1)$, and $\xi=$ $(a x y)^{\infty}$

14. $\varphi(a)=(a x y)^{l} a x, \varphi(x)=(y a x)^{m} y a, \varphi(y)=(x y a)^{n} x y(l, m, n \geq 0)$, and $\xi=(a x y)^{\infty}$;

15. $\varphi(a) \in w^{+}, \varphi(x y) \in w^{+}\left(w \in\{a, x y\}^{+}, a \triangleleft w, y \triangleleft \varphi(y)\right)$, and $\xi=w^{\infty}$;

16. $\varphi(a y) \in w^{+}, \varphi(x) \in w^{+}\left(w \in\{a y, x\}^{+}, a \triangleleft w, y \triangleleft \varphi(y)\right)$, and $\xi=w^{\infty}$;

17. $\varphi(a y) \in w^{+}, \varphi(x y) \in w^{+}\left(w \in\{a y, x y\}^{+}, a \triangleleft w, y \triangleleft \varphi(y)\right)$, and $\xi=w^{\infty}$;

18. $\varphi(a)=\left(a y^{m} x y^{n}\right)^{i} a, \varphi(x)=\left(x y^{n} a y^{m}\right)^{j} x, \varphi(y)=y(m, n \geq 0, i, j \geq 1)$, and $\xi=\left(a y^{m} x y^{n}\right)^{\infty}$

19. There exist two words $u=u_{1} \cdots u_{n} \in w^{+}$and $v=v_{1} \cdots v_{m} \in w^{+}$for some word $w=w_{1} \cdots w_{k} \in\{a, x\}^{+}$with $w_{1}=a$, and integers $\mathcal{P}(\alpha), \mathcal{D}(\alpha, \beta)$ and $\mathcal{S}(\alpha)$ for any $\alpha, \beta \in\{a, x\}$ satisfying the following two conditions:

(*) $\mathcal{P}(a)=0$; If $\beta \triangleright w$, then $\mathcal{S}(\beta)=0$;

(**) there is a constant $\mathcal{C}$, such that for any factor $\alpha \beta$ of length 2 of $w^{2}$, we have $\mathcal{S}(\alpha)+\mathcal{D}(\alpha, \beta)+\mathcal{P}(\beta)=\mathcal{C}$,

such that $\varphi(a)=u_{1} y^{\mathcal{D}\left(u_{1}, u_{2}\right)} \cdots y^{\mathcal{D}\left(u_{n-1}, u_{n}\right)} u_{n} y^{\mathcal{S}(a)}$,

$$
\begin{aligned}
& \varphi(x)=y^{\mathcal{P}(x)} v_{1} y^{\mathcal{D}\left(v_{1}, v_{2}\right)} \cdots y^{\mathcal{D}\left(v_{m-1}, u_{m}\right)} v_{m} y^{\mathcal{S}(x)}, \\
& \varphi(y)=y,
\end{aligned}
$$

and then $\xi=\left[w_{1} y^{\mathcal{D}\left(w_{1}, w_{2}\right)} \cdots y^{\mathcal{D}\left(w_{k-1}, w_{k}\right)} w_{n} y^{\mathcal{C}}\right]^{\infty}$;

20. $\varphi(a)=(a u)^{k} a, \varphi(x)=x, \varphi(y)=y\left(u \in\{x, y\}^{+}, k \geq 1\right)$, and $\xi=(a u)^{\infty}$;

21. $\varphi(a)=a u_{1} a \cdots a u_{k} a, \varphi(x)=x, \varphi(y)=x\left(u_{i} \in\{x, y\}^{+},\left|u_{1}\right|=\cdots=\left|u_{k}\right|=\right.$ $L, k \geq 1)$, and $\xi=\left(a u_{1} a \cdots a u_{k} a x^{L}\right)^{\infty}$.

In the last section, we give several remarks concerning the (possibly erasing) substitutions which fix a ultimately periodic sequence.

\section{Preliminaries}

In this paper, we shall use the following terminology. The readers can find more details in [5].

Let $w \in S^{*}$ be a word. We denote by $|w|$ the length of $w$, and, for a letter $s \in S$, by $|w|_{s}$ the number of occurrences of the letter $s$ in $w$. We denote by $\operatorname{Alph}(w)$ the set of letters appearing in $w$, that is $\operatorname{Alph}(w)=\left\{s \in S:|w|_{s} \geq 1\right\}$. In particular, $\operatorname{Alph}(\varepsilon)=\emptyset$.

A word $u \in S^{+}$is said to be primitive if it is not a power of another word, that is, the condition $u=v^{k}$ for some $v \in S^{+}$implies $k=1$. Any word is a power of a primitive word. 
Two words $u$ and $v$ are said to be conjugate if there exist words $x, y \in S^{*}$ such that $u=x y$ and $v=y x$. In this case, we also say that $v$ is a conjugate word of $u$.

The next facts are basic results in Combinatorics on Words (see below for the definition of $w^{*}$ ).

Lemma 1.1. Let $w$ be a primitive word, $u$ be a word. Then

1. if $u$ and $w$ are conjugate, then $u$ is also primitive;

2. if $u w \prec w^{k}$ for some $k$, then $u \triangleright w^{k}$; if $w u w \prec w^{k}$, then $u \in w^{*}$;

3. $u \in w^{*} \Longleftrightarrow u^{k} \in w^{*}$ for some $k \geq 1 \Longleftrightarrow u^{k} \in w^{*}$ for any $k$.

The following theorem is classical in the study of periodicity [1].

Theorem 1.2 (Fine and Wilf). Let $x, y \in S^{*}, n=|x|, m=|y|, d=\operatorname{gcd}(n, m)$. If two powers $x^{p}$ and $y^{q}$ of $x$ and $y$ have a common prefix of length at least equal to $n+m-d$, then there is a word $w$ such that $x, y \in w^{*}$.

Let $\xi \in S^{\mathbb{N}}$ be a sequence. We call $\xi$ periodic if there exists a word $w$ such that $\xi=w^{\infty}(:=w w w \cdots)$; we call $\xi$ ultimately periodic if there exist words $u$ and $w$ such that $\xi=u w^{\infty}$.

Let $\xi$ be a sequence. The language of length $n$ of $\xi$, denoted by $L_{n}(\xi)$, is the set of all factors of length $n$ occurring in $\xi$. We call complexity function of $\xi$, denoted by $p_{\xi}(n)$, the function which with each positive integer $n$ associated the number $\# L_{n}(\xi)$, where \# denotes the cardinality of a finite set. A factor $w$ of $\xi$ is called a (right) special factor if it has more than one right extensions, that is, there are different letters $x$ and $y$ such that both $w x$ and $w y$ are factors.

The following lemma is elementary.

Lemma 1.3. Let $\xi$ be a sequence. The following are equivalent:

1. the sequence $\xi$ is ultimately periodic;

2. the complexity function of $\xi$ is bounded;

3. the lengths of the special factors of $\xi$ are bounded.

Let $\xi$ be a sequence. We say that $\xi$ is recurrent if every factor of $\xi$ occurs for infinitely many times. If $\xi$ is recurrent, then $\xi$ is ultimately periodic if and only if it is periodic.

Given a nonempty subset $X$ of the free monoid $S^{*}$, we denote by $X^{*}$ the submonoid of $S^{*}$ generated by $X$ (for simplification, we will write $w^{*}$ instead of $\{w\}^{*}$ for $w \in S^{+}$). Conversely, given a submonoid $P$ of $S^{*}$, there exist a unique set $X$ that generates $P$ and is minimal for set-inclusion.

Also, we put $X^{+}=X^{*} \backslash\{\varepsilon\}$ and $w^{+}=w^{*} \backslash\{\varepsilon\}$.

A monoid $M$ is said to be free if there exist an alphabet $A$ and an isomorphism of the free monoid $A^{*}$ onto $M$. For example, for any $w \in S^{+}, w^{*}$ is free.

Proposition 1.4 ([5], p. 5). Let $P$ be a submonoid of $S^{*}$ and $X$ be its minimal generating set. Then $P$ is free if and only if any equality

$$
x_{1} x_{2} \cdots x_{m}=y_{1} y_{2} \cdots y_{n}, \quad n, m \geq 0, x_{i}, y_{j} \in X
$$

implies $n=m$ and $x_{i}=y_{i}, 1 \leq i \leq n$. 
The minimal generating set of a free submonoid $P$ of $S^{*}$ is called a code. the intersection of all free submonoids of $S^{*}$ containing $X$ is the smallest free submonoid containing $X$; the code generating this submonoid is called the free hull of $X$.

Theorem 1.5 (defect theorem, [5], p. 6). The free hull $Y$ of a finite subset $X \subset S^{*}$, which is not a code, satisfies the inequality

$$
\# Y \leq \# X-1
$$

Let $\varphi$ be a substitution of $S^{*}$. A letter $s \in S$ is growing (for $\varphi$ ) if $\left\{\left|\varphi^{n}(s)\right|: n \geq 1\right\}$ is unbounded; otherwise the letter $s$ will be called a bounded letter.

A substitution $\varphi$ is called cyclic if there is a word $w \in S^{+}$such that for every letter $s \in S, \varphi(s) \in w^{*}$. And $\varphi$ is called ultimately cyclic if some power $\varphi^{n}$ is cyclic. It is easy to see that the sequence generated by a ultimately cyclic substitution is periodic.

Let $\varphi: S^{*} \rightarrow S^{*}$ be a substitution. It is simplifiable if there exist an alphabet $X$, \#X<\#S and two morphisms $\sigma: S^{*} \rightarrow X^{*}$, and $\tau: X^{*} \rightarrow S^{*}$ such that $\varphi=\tau \circ \sigma$. And $\varphi$ is elementary if it is not simplifiable.

If a substitution $\varphi$ is elementary, then it is injective over $S^{*}$ and the set $\{\varphi(s)$ : $s \in S\}$ is a code ([7], p. 131).

\section{Periodicity Problem over A three-Letter Alphabet}

Now we will concentrate on the case of the three-letter alphabet. We always use $S=\{a, b, c\}$ to denote the alphabet. The notation $\varphi=(u, v, w)$ denotes the substitution $\varphi(a)=u, \varphi(b)=v, \varphi(c)=w$. In this section, we find all substitutions over $S$ which generate a ultimately periodic sequence.

Hereafter we assume that $\varphi$ is a substitution over $S$ which, with the axiom $a$, generates the sequence $\xi=\varphi^{\infty}(a)$, and $\xi$ is ultimately periodic.

According as whether each letter appears in the sequence $\xi$ for infinitely many times, we consider two cases.

\subsection{The SEQUence $\xi$ CONTAINS SOME LETTER For OnLY Finite times}

Suppose that $\xi$ contains some letter for only finite times, then, writing

$$
\xi=u w^{\infty} \quad \text { with } u \in S^{*}, w \in S^{+},
$$

we have

$$
1 \leq \# \operatorname{Alph}(w) \leq 2 .
$$

There are two possibilities:

Case I. \# $\operatorname{Alph}(w)=1$.

Without loss of generality, we take $w=x \in S$. And thus $\varphi(x) \in x^{+}$. 
Subcase I.1. $x=a$.

Then the substitution $\varphi$ is of the form:

- $\varphi(a)=a^{k}, \varphi(b), \varphi(c) \in S^{+}, k \geq 2$, and $\xi=a^{\infty}$.

Subcase I.2. $x \neq a$.

In this subcase, $\varphi(a)=a v, a \notin \operatorname{Alph}(v)$ (otherwise, the sequence $\xi$ contains $a$ for infinite times).

Let $y$ be the letter such that $\{a, x, y\}=\{a, b, c\}$. If $y \in \operatorname{Alph}(v)$, then $\varphi(y) \in x^{+}$ (otherwise, the letter $y$ occurs for infinitely many times in $\xi$ ).

Therefore, the substitution $\varphi$ is of one of the following forms:

- $\varphi(a)=a x^{k}, \varphi(x) \in x^{+}, \varphi(y) \in S^{+}, k \geq 1$, and $\xi=a x^{\infty}$;

- $\varphi(a)=a v, \varphi(x) \in x^{+}, \varphi(y) \in x^{+}, v \in\{x, y\}^{+}$, and $\xi=a v x^{\infty}$.

Case II. $\# \operatorname{Alph}(w)=2$. Let $x, y \in S \operatorname{such}$ that $\operatorname{Alph}(w)=\{x, y\}$. Then $\varphi(x), \varphi(y) \in\{x, y\}^{+}$.

Subcase II.1. $a \in\{x, y\}$.

In this subcase, the restriction $\left.\varphi\right|_{\{x, y\}^{*}}$ of $\varphi$ to $\{x, y\}^{*}$ generates the sequence $\xi$, and thus (with $z \in S$ such that $\{x, y, z\}=\{a, b, c\}$ ):

- $\left.\varphi\right|_{\{x, y\}^{*}}$ is one of the forms in Theorem $0.1, \varphi(z) \in S^{+}$.

Subcase II.2. $a \notin\{x, y\}$.

In this subcase, we have $\varphi(a)=a v$ with $v \in\{x, y\}^{+}$(in fact, as in Subcase I.2, $a \in \operatorname{Alph}(v)$ implies that $a$ appears infinitely often).

Subsubcase II.2.1. Suppose both $x$ and $y$ are bounded. There are 3 possibilities:

(i) $\varphi(x)=x, \varphi(y)=y$. Then $\xi=a v^{\infty}$;

(ii) $\varphi(x)=y, \varphi(y)=x$. Then $\xi=a(v \varphi(v))^{\infty}$;

(iii) $\varphi(x)=\varphi(y) \in\{x, y\}$. In this case, without loss of generality, suppose $\varphi(x)=x$, and thus $\xi=\varphi^{\infty}(a)=a v x^{\infty}$. The sequence $\xi$ contains the letter $y$ for only finite times, and it is a contradiction.

So, in this subsubcase, the substitution $\varphi$ is of one of the following forms:

- $\varphi(a)=a v, \varphi(x)=x, \varphi(y)=y, v \in\{x, y\}^{+}$, and $\xi=a v^{\infty}$

- $\varphi(a)=a v, \varphi(x)=y, \varphi(y)=x, v \in\{x, y\}^{+}$, and $\xi=a(v \varphi(v))^{\infty}$.

Subsubcase II.2.2. Suppose either $x$ or $y$ is growing. Then the substitution $\left.\varphi^{2}\right|_{\{x, y\}} *$ generates an infinite sequence $\eta$. Without loss of generality, we assume that $\eta=\left(\left.\varphi^{2}\right|_{\{x, y\}^{*}}\right)^{\infty}(x)$. It is easy to see that all factors of $\eta$ are factors of $\xi$, thus $\eta$ is also ultimately periodic. Then by Theorem 0.1 , and noticing that both $x$ and $y$ occur in $\eta$ for infinitely many times, there are 3 possibilities:

(i) $\varphi^{2}(x) \in t^{+}$and $\varphi^{2}(y) \in t^{+}$for some $t \in\{x, y\}^{+}$.

In this case, we have that $\{\varphi(x), \varphi(y)\}$ is not a code. Then by defect theorem (Th. 1.5), there is a word $r \in\{x, y\}^{+}$such that $\varphi(x), \varphi(y) \in r^{+}$, and thus

- $\varphi(a)=a v, \varphi(x) \in r^{+}, \varphi(y) \in r^{+}, v, r \in\{x, y\}^{+}$, and $\xi=a v r^{\infty}$.

(ii) $\varphi^{2}(x)=(x y)^{i} x$ and $\varphi^{2}(y)=(y x)^{j} y$.

In this case we have either $\varphi(x)=(x y)^{l} x, \varphi(y)=(y x)^{m} y$ or $\varphi(x)=y(x y)^{l}$, $\varphi(y)=x(y x)^{m}$. 
Since $\xi=\varphi^{\infty}(a)=a v \varphi(v) \varphi^{2}(v) \cdots=t(x y)^{\infty}$ for some word $t$, then for $k$ large enough, we have $\varphi^{k}(v) \prec(x y)^{\infty}$, hence $v \prec(x y)^{\infty}$.

Without loss of generality, we suppose $x \triangleleft v$. An easy discussion gives that $\varphi$ is of one of the following forms:

- $\varphi(a)=a(x y)^{n}, \varphi(x)=(x y)^{l} x, \varphi(y)=(y x)^{m} y, l, n \geq 1, m \geq 0$, and $\xi=a(x y)^{\infty}$

- $\varphi(a)=a(x y)^{n} x, \varphi(x)=y(x y)^{l}, \varphi(y)=x(y x)^{m}, l \geq 1, m, n \geq 0$, and $\xi=a(x y)^{\infty}$.

(iii) $\varphi^{2}(x)=\left(x y^{p}\right)^{q} x$ and $\varphi^{2}(y)=y$.

Then we have that $\varphi(x)=\left(x y^{l}\right)^{m} x, \varphi(y)=y$. In this case, the word $v$ is a conjugate word of some power of $\left(x y^{l}\right)$, and thus

- $\varphi(a)=a\left(y^{i} x y^{l-i}\right)^{n}, \varphi(x)=\left(x y^{l}\right)^{m} x, \varphi(y)=y, l, m, n \geq 1,0 \leq i \leq l$, and $\xi=a y^{i}\left(x y^{l}\right)^{\infty}$.

Up to now, we have shown:

Theorem 2.1. Let $\varphi$ be a substitution over $S$. Suppose that $\varphi$ generates, with the axiom a, a sequence $\xi$, and $\xi=u w^{\infty}$ with $1 \leq \# \operatorname{Alph}(w) \leq 2$. Then there is a permutation $(x, y)$ of the letters $(b, c)$ such that $\varphi$ has one of the following forms:

(i) $\varphi(a)=a^{k}, \varphi(x) \in S^{+}, \varphi(y) \in S^{+}(k \geq 2)$, and $\xi=a^{\infty}$;

(ii) $\varphi(a)=a x^{k}, \varphi(x) \in x^{+}, \varphi(y) \in S^{+}(k \geq 1)$, and $\xi=a x^{\infty}$;

(iii) $\varphi(a)=(a v)^{p}, \varphi(x)=(a v)^{q}, \varphi(y) \in S^{+}\left(v \in\{a, x\}^{+}, p, q \geq 1\right)$, and $\xi=$ $(a v)^{\infty}$

(iv) $\varphi(a)=(a x)^{p} a, \varphi(x)=(x a)^{q} x, \varphi(y) \in S^{+}(p, q \geq 1)$, and $\xi=(a x)^{\infty}$;

(v) $\varphi(a)=\left(a x^{p}\right)^{q} a, \varphi(x)=x, \varphi(y) \in S^{+}(p, q \geq 1)$, and $\xi=\left(a x^{p}\right)^{\infty}$;

(vi) $\varphi(a)=a v, \varphi(x)=x, \varphi(y)=y\left(v \in\{x, y\}^{+}\right)$, and $\xi=a v^{\infty}$;

(vii) $\varphi(a)=a v, \varphi(x)=y, \varphi(y)=x\left(v \in\{x, y\}^{+}\right)$, and $\xi=a(v \varphi(v))^{\infty}$;

(viii) $\varphi(a)=a v, \varphi(x) \in r^{+}, \varphi(y) \in r^{+}\left(v, r \in\{x, y\}^{+}\right)$, and $\xi=a v r^{\infty}$;

(ix) $\varphi(a)=a(x y)^{n}, \varphi(x)=(x y)^{l} x, \varphi(y)=(y x)^{m} y(l, n \geq 1, m \geq 0)$, and $\xi=$ $a(x y)^{\infty}$;

(x) $\varphi(a)=a(x y)^{n} x, \varphi(x)=y(x y)^{l}, \varphi(y)=x(y x)^{m}(l \geq 1, m, n \geq 0)$, and $\xi=a(x y)^{\infty}$;

(xi) $\varphi(a)=a\left(y^{i} x y^{l-i}\right)^{n}, \varphi(x)=\left(x y^{l}\right)^{m} x, \varphi(y)=y(l, m, n \geq 1,0 \leq i \leq l)$, and $\xi=a y^{i}\left(x y^{l}\right)^{\infty}$.

Remark that the second case in Subcase I.2 and (i) in Subsubcase II.2.2 is summarized into case (viii) in the above theorem.

\subsection{The SEQUENCE $\xi$ CONTAINS EACH LETTER FOR INFINITE TIMES}

In this subsection, we suppose that the sequence $\xi$ contains each letter for infinite times. The study is divided into two parts, depending on whether the substitution is ultimately cyclic or not. 


\subsubsection{Ultimately cyclic substitution}

As mentioned before, the sequence generated by a ultimately cyclic substitution is periodic. In this subsection we study the ultimately cyclic substitutions.

In the binary alphabet case, we have

Proposition 2.2. Let $\varphi$ be a substitution over a two-letter alphabet, then $\varphi$ is ultimately cyclic if and only if $\varphi$ is cyclic.

Proof. Denote the alphabet $S=\{a, b\}$. Suppose that $\varphi$ is ultimately cyclic and let $n$ be the minimal integer such that $\varphi^{n}$ is cyclic.

Assume $n>1$, then we have $\varphi^{n}(a b)=\varphi^{n}(b a)$ and $\varphi^{n-1}(a b) \neq \varphi^{n-1}(b a)$. Since $\varphi\left(\varphi^{n-1}(a b)\right)=\varphi\left(\varphi^{n-1}(b a)\right)$, by Theorem 1.4 , we know that $\{\varphi(a), \varphi(b)\}$ is not a code. By defect theorem (Th. 1.5), $\varphi(a), \varphi(b) \in w^{*}$ for some word $w$. Hence $\varphi^{n-1}(a b)=\varphi^{n-1}(b a)$. This is a contradiction.

So $n=1$ and $\varphi$ itself is cyclic.

In the ternary alphabet case, the above theorem does not hold. For example, $(a c, a c b, b)^{2}=(a c b, a c b a c b, a c b)$. But a little weaker result holds.

Theorem 2.3. Let $\varphi$ be a substitution over a three-letter alphabet, then $\varphi$ is ultimately cyclic if and only if $\varphi^{2}$ is cyclic.

Proof. Write $S=\{a, b, c\}$ and suppose $\varphi^{n}$ is cyclic over $S$.

In the same way as in the proof of the above theorem, we show that $\{\varphi(a), \varphi(b)$, $\varphi(c)\}$ is not a code. Denote by $Y$ the free hull of $\{\varphi(a), \varphi(b), \varphi(c)\}$. By defect theorem (Th. 1.5), $\# Y \leq 2$.

If $\# Y=1$, then $\varphi$ is cyclic, thus $\varphi^{2}$ is also cyclic.

If $Y=\{u, v\}$. Let us introduce a new two-letter alphabet $X=\{A, B\}$ and define two morphisms as follows: For each letter $s \in S, \varphi(s) \in Y^{*}$. Since $Y=$ $\{u, v\}$ is a code, $\varphi(s)$ has a unique decomposition $\varphi(s)=w_{1} w_{2} \cdots w_{m} \in Y^{m}\left(w_{i} \in\right.$ $Y)$. Then define the morphism $\psi: S^{*} \rightarrow X^{*}$ as $\psi(s)=C_{1} C_{2} \cdots C_{m} \in X^{m}$, where $C_{i}=A$ if $w_{i}=u$ and $C_{i}=B$ if $w_{i}=v$. Another morphism $\pi: X^{*} \rightarrow S^{*}$ is defined as $\pi(A)=u$ and $\pi(B)=v$.

Then $\varphi=\pi \circ \psi$. And $\Phi:=\psi \circ \pi$ defines a substitution over $X^{*}$.

Since $\varphi^{n}$ is cyclic, there is a word $w \in S^{*}$ such that $\varphi^{n}(s) \in w^{*}$ for any $s \in S$. Thus for any letter $x \in X, \varphi^{n}(\pi(x)) \in w^{*}$, hence

$$
\begin{aligned}
\Phi^{n+1}(x) & =(\psi \circ \pi)^{n+1}(x)=\psi \circ(\pi \circ \psi)^{n} \circ \pi(x) \\
& =\psi \circ \varphi^{n} \circ \pi(x)=\psi\left(\varphi^{n}(\pi(x))\right) \\
& \in \psi\left(w^{*}\right)=(\psi(w))^{*} .
\end{aligned}
$$

Since $\psi(w)$ is a word in $X^{*}$, this implies that $\Phi$ is ultimately cyclic over the alphabet $X$. By Proposition 2.2, $\Phi$ is cyclic, i.e. there is a word $W \in X^{*}$ such that $\Phi(x) \in W^{*}$ for any $x \in X$, and thus for any letter $s \in S, \Phi(\psi(s)) \in W^{*}$. Hence

$$
\varphi^{2}(s)=\pi \circ \psi \circ \pi \circ \psi(s)=\pi(\Phi(\psi(s))) \in \pi\left(W^{*}\right)=(\pi(W))^{*},
$$

this is to say that $\varphi^{2}$ is cyclic. 
Now an induction on $n$ gives the following result.

Proposition 2.4. A substitution $\varphi$ over an n-letter alphabet is ultimately cyclic if and only if $\varphi^{n-1}$ is cyclic.

\subsubsection{Non-ultimately cyclic substitution}

Now we turn to study the non-ultimately cyclic substitutions

When $\xi$ contains each letter for infinite times, it is recurrent. Recall that if a sequence is recurrent and ultimately periodic, then it is periodic. Therefore, in the subsection, we will always write that $\xi=w^{\infty}$ with $w$ a primitive word and $\operatorname{Alph}(w)=\{a, b, c\}$.

We will consider three cases according to the number of growing letters. Recalling that $\varphi(a)=a v$ with $v \in S^{+}$, the letter $a$ is growing.

Case I. All letters are growing.

Theorem 2.5. Let $\varphi$ be a non-ultimately cyclic substitution over S. Suppose that $\varphi$ generates, with the axiom a, a periodic sequence $\xi$, and $\xi$ contains each letter for infinite times. If any letter is growing, then there is a permutation $(x, y)$ of the letters $(b, c)$ such that $\varphi$ has one of the following forms:

(i) $\varphi(a)=(a x y)^{l} a, \varphi(x)=(x y a)^{m} x, \varphi(y)=(y a x)^{n} y(l, m, n \geq 1)$, and $\xi=$ $(\text { axy })^{\infty}$;

(ii) $\varphi(a)=(a x y)^{l} a x, \varphi(x)=(y a x)^{m} y a, \varphi(y)=(x y a)^{n} x y(l, m, n \geq 0)$, and $\xi=(a x y)^{\infty}$;

(iii) $\varphi(a) \in w^{+}, \varphi(x y) \in w^{+}\left(w \in\{a, x y\}^{+}, a \triangleleft w, y \triangleleft \varphi(y)\right)$, and $\xi=w^{\infty}$;

(iv) $\varphi(a y) \in w^{+}, \varphi(x) \in w^{+}\left(w \in\{a y, x\}^{+}, a \triangleleft w, y \triangleleft \varphi(y)\right)$, and $\xi=w^{\infty}$;

(v) $\varphi(a y) \in w^{+}, \varphi(x y) \in w^{+}\left(w \in\{a y, x y\}^{+}, a \triangleleft w, y \triangleleft \varphi(y)\right)$, and $\xi=w^{\infty}$.

Remark that, in above theorem, the substitutions of types (i) and (ii) are elementary, while the ones of the last three types are simplifiable. Also, the proof will be divided into two parts.

First we consider the elementary substitutions. The following lemma comes from $[6]$.

Lemma 2.6. Let $\varphi$ be an elementary substitution. Then $\varphi$ generates a ultimately periodic sequence $\xi$ if and only if $\xi$ has no special word of form $x u$, where $x$ is a growing letter and $u$, possibly empty, is a word consisting of bounded letters.

Proof for the elementary cases in Theorem 2.5. If $\varphi$ is an elementary substitution and every letter is growing, then $\varphi$ generates a periodic sequence $\xi$ if and only if $\xi$ has no special words. In this case, $\xi=(a x y)^{\infty}$ for some permutation $(x, y)$ of the letters $(b, c)$. From here we can derive easily the type of $\varphi$.

In fact, since $\varphi$ is elementary, we get that $\varphi$ is of one of the following forms:

- $\varphi(a)=(a x y)^{l} a, \varphi(x)=(x y a)^{m} x, \varphi(y)=(y a x)^{n} y(l, m, n \geq 1)$, and $\xi=(a x y)^{\infty}$

- $\varphi(a)=(a x y)^{l} a x, \varphi(x)=(y a x)^{m} y a, \varphi(y)=(x y a)^{n} x y(l, m, n \geq 0)$, and $\xi=(a x y)^{\infty}$. 
These are just the cases (i) and (ii) in Theorem 2.5.

Let us consider the simplifiable substitutions.

Proof for the simplifiable cases in Theorem 2.5. Suppose that $\varphi$ is simplifiable, and $Y$ is the free hull of $\{\varphi(a), \varphi(b), \varphi(c)\}$. Then $\# Y \leq 2$.

Since $\# Y=1$ implies that $\varphi$ is cyclic, we only need consider the case that $\# Y=2$.

Take $n$ large enough such that $\left|\varphi^{n-1}(s)\right|>|w|$ for any letter $s \in S$. Since $\{\varphi(a), \varphi(b), \varphi(c)\} \subset Y^{*}$, by Pigeon hole principle, at least two of $\{\varphi(a), \varphi(b), \varphi(c)\}$ have the same first letter. Denote the two words by $\varphi(x)$ and $\varphi(y)$, and the same first letter by $\alpha$. Hence $\varphi^{n-1}(\alpha)$ is the prefix of both $\varphi^{n}(x)$ and $\varphi^{n}(y)$, and the length of $\varphi^{n-1}(\alpha)$ is larger than $|w|$. Since $\varphi^{n}(x), \varphi^{n}(y) \prec w^{\infty}$, there exists a conjugate word $w_{1}$ of $w$ such that $\varphi^{n}(x)=w_{1}^{l} r, \varphi^{n}(y)=w_{1}^{m} t$ with $l, m \geq 1$ and $r, t$ proper prefixes of $w_{1}$.

Recalling that $x, y$ and $z$ occur infinitely often, at least one of $z x$ or $z y$ is a factor of $\xi, \varphi^{n}(z) w_{1} \prec \xi$, and thus $\varphi^{n}(z) w_{1} \prec w_{1}^{\infty}$. By Lemma 1.1, we can write $\varphi^{n}(z)=u w_{1}^{p}$ with $p \geq 1$ and $u$ a proper suffix of $w_{1}$. So we have

$$
\varphi^{n}(x)=w_{1}^{l} r, \varphi^{n}(y)=w_{1}^{m} t, \varphi^{n}(z)=u w_{1}^{p} \text { with } l, m, p \geq 1 \text { and } r, t \triangleleft w_{1}, u \triangleright w_{1} .
$$

Subcase I.1 Either $r$ or $t$, say $r$, is empty.

If $x z$ is a factor, then $u=\varepsilon$, and the fact that one of $y x$ and $y z$ is a factor implies $t=\varepsilon$, thus $\varphi$ is ultimately cyclic.

If $z z$ is a factor, then $u=\varepsilon$ and $\varphi$ is ultimately cyclic.

If either $y x$ or $y y$ is a factor, then $t=\varepsilon$ and $\varphi$ is ultimately cyclic.

Thus if $\varphi$ is non-ultimately cyclic, then $L_{2}(\xi) \subset\{x x, x y, y z, z x, z y\}$. Moreover we claim that $w_{1} \in\{x, y z\}^{*}$.

In fact, suppose $z \triangleleft w_{1}$. Since $\varphi$ generates an infinite sequence, $z \triangleleft \varphi(z)$, thus $\varphi^{n}(z) \triangleleft w_{1}^{\infty}$, and thus $u=\varepsilon$ and $\varphi$ is ultimately cyclic.

Since $z$ is not a prefix of $w_{1}$, and neither $y x$ nor $y y$ is a factor, then $y$ is not a suffix of $w_{1}$ (otherwise either $y x \prec w_{1}^{2}$ or $y y \prec w_{1}^{2}$ ). Thus, by $L_{2}(\xi) \subset$ $\{x x, x y, y z, z x, z y\}$, we have $w_{1} \in\{x, y z\}^{*}$. The claim follows.

Hence $\varphi^{n}(x), \varphi^{n}(y z) \in w_{1}^{*}$ with $w_{1} \in\{x, y z\}^{*}$.

Now $\varphi^{n}(x)$ and $\varphi^{n}(y)$ have the same prefix as $w_{1}$, but $\varphi^{n}(z)$ has a different prefix (otherwise $\varphi$ will be ultimately cyclic). By enumerating all the possible cases, we show that the first letter of both $\varphi(x)$ and $\varphi(y)$ is either $x$ or $y$, and the last letter of both $\varphi(x)$ and $\varphi(z)$ is either $x$ or $z$. Moreover $y \triangleright \varphi(y)$ and $z \triangleleft \varphi(z)$. Thus $\varphi(x), \varphi(y z) \in\{x, y z\}^{*}$.

We can look the substitution $\varphi$ as a substitution over the alphabet $\{x, y z\}$ (with a slight abuse of notation), and $\varphi$ is ultimately cyclic over this alphabet. By Proposition $2.2, \varphi$ is cyclic over this alphabet, i.e. we have that

- $\varphi(x) \in w^{+}, \varphi(y z) \in w^{+}$with $w \in\{x, y z\}^{+}, z \triangleleft \varphi(z)$, and $\xi=w^{\infty}$.

This corresponds to the cases (iii) and (iv) in Theorem 2.5. 
Subcase I.2 Neither $r$ nor $t$ is empty.

If either $x x$ or $x y$ is a factor, $r=\varepsilon$; if either $y x$ or $y y$ is a factor, $t=\varepsilon$; if $z z$ is a factor, then $u=\varepsilon$, and (since one of $x x, x y$ and $x z$ is a factor) $r=\varepsilon$.

Thus, in this case, we have $L_{2}(\xi)=\{x z, y z, z x, z y\}$.

As in case Subcase I.1, we have that either $x \triangleleft w_{1}$ or $y \triangleleft w_{1}$, and $\xi=w_{1}^{\infty}$. Moreover $\varphi^{n}(x z), \varphi^{n}(y z) \in w_{1}^{*}$ with $w_{1} \in\{x z, y z\}^{*}$.

Similar to case Subcase I.1, $\varphi(x z), \varphi(y z) \in\{x z, y z\}^{*}$ and $\varphi$ is a ultimately cyclic substitution over the alphabet $\{x z, y z\}, z \triangleleft \varphi(z)$. Thus

- $\varphi(x z) \in w^{+}, \varphi(y z) \in w^{+}$with $w \in\{x z, y z\}^{+}, z \triangleleft \varphi(z)$, and $\xi=w^{\infty}$.

This corresponds to the case (v) in Theorem 2.5.

Case II. One and only one letter is bounded.

Theorem 2.7. Let $\varphi$ be a non-ultimately cyclic substitution over $S$. Suppose that $\varphi$ generates, with the axiom a, a periodic sequence $\xi$, and $\xi$ contains any letter for infinite times. If one letter is bounded and other letters are growing for $\varphi$, then there is a permutation $(x, y)$ of the letters $(b, c)$ such that $\varphi$ has one of the following forms:

(i) $\varphi(a)=\left(a y^{m} x y^{n}\right)^{i} a, \varphi(x)=\left(x y^{n} a y^{m}\right)^{j} x, \varphi(y)=y(m, n \geq 0, i, j \geq 1)$, and $\xi=\left(a y^{m} x y^{n}\right)^{\infty}$;

(ii) there exist two words $u=u_{1} \cdots u_{n} \in w^{+}$and $v=v_{1} \cdots v_{m} \in w^{+}$for some word $w=w_{1} \cdots w_{k} \in\{a, x\}^{+}$with $w_{1}=a$, and integers $\mathcal{P}(\alpha), \mathcal{D}(\alpha, \beta)$ and $\mathcal{S}(\alpha)$ for any $\alpha, \beta \in\{a, x\}$ satisfying the following two conditions:

(*) $\mathcal{P}(a)=0$; If $\beta \triangleright w$, then $\mathcal{S}(\beta)=0$;

(**) there is a constant $\mathcal{C}$, such that for any factor $\alpha \beta$ of length 2 of $w^{2}$, we have

such that

$$
\mathcal{S}(\alpha)+\mathcal{D}(\alpha, \beta)+\mathcal{P}(\beta)=\mathcal{C}
$$

$$
\begin{aligned}
\varphi(a) & =u_{1} y^{\mathcal{D}\left(u_{1}, u_{2}\right)} \cdots y^{\mathcal{D}\left(u_{n-1}, u_{n}\right)} u_{n} y^{\mathcal{S}(a)}, \\
\varphi(x) & =y^{\mathcal{P}(x)} v_{1} y^{\mathcal{D}\left(v_{1}, v_{2}\right)} \cdots y^{\mathcal{D}\left(v_{m-1}, u_{m}\right)} v_{m} y^{\mathcal{S}(x)}, \\
\varphi(y) & =y
\end{aligned}
$$

and then $\xi=\left[w_{1} y^{\mathcal{D}\left(w_{1}, w_{2}\right)} \cdots y^{\mathcal{D}\left(w_{k-1}, w_{k}\right)} w_{n} y^{\mathcal{C}}\right]^{\infty}$.

Proof. We denote by $y$ the bounded letter, and by $x$ the growing letter in $\{b, c\}$. Then $\varphi(y)=y$.

First, we have

Claim 1. Fixed $\alpha, \beta \in\{a, x\}$, then all the powers of $y$ between the letter $\alpha$ and $\beta$ are the same, i.e. if $\alpha y^{i} \beta \prec \xi$ and $\alpha y^{j} \beta \prec \xi$, then $i=j$.

In fact, since both $\alpha$ and $\beta$ are growing and $\xi=w^{\infty}$, there exists $n$ such that $\varphi^{n}(\alpha)=d w^{p} e$ and $\varphi^{n}(\beta)=f w^{q} g$ with $d, f$ proper prefixes of $w, e, g$ proper suffixes of $w$; and $p, q \geq 1$. Since $\varphi^{n}\left(\alpha y^{i} \beta\right)$ and $\varphi^{n}\left(\alpha y^{j} \beta\right)$ are factors, we have $w e y^{i} f w, w e y^{j} f w \prec \xi=w^{\infty}$. Since the word $w$ is primitive, we have, by Lemma 1.1, that $e y^{i} f, e y^{j} f \in w^{*}$. And, noticing that $w \notin y^{*}$, this implies that $i=j$. Claim 1 follows.

Define a morphism $\pi:\{a, x, y\}^{*} \rightarrow\{a, x\}^{*}$ as $\pi(a)=a, \pi(x)=x$, and $\pi(y)=\varepsilon$. Then for $w \in\{a, x, y\}^{*}$, the word $\pi(w)$ is obtained by erasing all the $y$ 's from $w$. 
The substitution $\varphi$ induces a substitution $\theta$ over the alphabet $\{a, x\}$ as follows: $\theta(a)=\pi(\varphi(a))$ and $\theta(x)=\pi(\varphi(x))$.

Since $\varphi(y)=y$, we know that $\theta$ generates, with the axiom $a$, the sequence $\pi(\xi)=\theta^{\infty}(a)$. Thus by the periodicity of $\xi$, the sequence $\pi(\xi)$ is also periodic. Then by Theorem 0.1 , we can get the form of the substitution $\theta$. The rest thing is deriving the substitution $\varphi$ from $\theta$. There are three cases:

Subcase II.1. $\theta(a) \in u^{+}, \theta(x) \in u^{+}$with $u \in\{a, x\}^{+}$.

For $\alpha, \beta \in\{a, x\}$, define the integer $\mathcal{D}(\alpha, \beta)$ as follows: if $\alpha y^{k} \beta$ is a factor of $\xi$, then $\mathcal{D}(\alpha, \beta)=k$; if for any $k, \alpha y^{k} \beta$ is not a factor of $\xi$, then $\mathcal{D}(\alpha, \beta)=-1$.

For $\alpha \in\{a, x\}, \mathcal{P}(\alpha)$ (resp. $\mathcal{S}(\alpha)$ ) is defined as the maximal integer $k$ such that $y^{k} \triangleleft \varphi(\alpha)$ (resp. $\left.y^{k} \triangleright \varphi(\alpha)\right)$.

So $\mathcal{P}(a)=0$. And if $\beta \triangleright u$, then

$$
\mathcal{S}(\beta)=0
$$

(Indeed, if $y^{k} \triangleright \varphi(\beta)$, then $y^{n k} \triangleright \varphi^{n}(\beta)$ for any $n$. And thus $y^{n k}$ is a factor of $\xi$ for any $n$, while this is possible only if $k=0$.)

Moreover, we have

Claim 2. There is a constant $\mathcal{C}$ such that, for any $\alpha, \beta \in\{a, x\}$ with $\mathcal{D}(\alpha, \beta) \geq 0$,

$$
\mathcal{S}(\alpha)+\mathcal{D}(\alpha, \beta)+\mathcal{P}(\beta)=\mathcal{C}
$$

In fact, for $\alpha \in\{a, x\}$, since $\theta(\alpha) \in u^{+}$,

$$
\varphi(\alpha)=y^{\mathcal{P}(\alpha)} r t_{\alpha} s y^{\mathcal{S}(\alpha)}
$$

where $r \in\{a, x\}$ (resp. $s \in\{a, x\}$ ) is the first letter (resp. the last letter) of $u$, and $t_{\alpha}$ is a word depending on $\alpha$.

If $\mathcal{D}(\alpha, \beta) \geq 0$, then $\alpha y^{\mathcal{D}(\alpha, \beta)} \beta \prec \xi$, and $\varphi\left(\alpha y^{\mathcal{D}(\alpha, \beta)} \beta\right) \prec \xi$, hence $s y^{\mathcal{S}(\alpha)+\mathcal{D}(\alpha, \beta)+\mathcal{\mathcal { P }}(\beta)} r \prec \xi$. Recall that, for $\alpha y^{i} \beta$ and $\alpha y^{j} \beta$ factors of $\xi, i=j$. So there is a constant $\mathcal{C}$ such that the condition $s y^{k} r \prec \xi$ implies $k=\mathcal{C}$. And then $\mathcal{S}(\alpha)+\mathcal{D}(\alpha, \beta)+\mathcal{P}(\beta)=\mathcal{C}$. Claim 2 follows.

Now, if the numbers $\mathcal{D}, \mathcal{P}$ and $\mathcal{S}$ are known, we can rebuild $\varphi(a)$ and $\varphi(x)$ from $\theta(a)$ and $\theta(x)$. In fact, if $\theta(\alpha)=u_{1} \cdots u_{n}$, then

$$
\varphi(\alpha)=y^{\mathcal{P}(\alpha)} u_{1} y^{\mathcal{D}\left(u_{1}, u_{2}\right)} \cdots y^{\mathcal{D}\left(u_{n-1}, u_{n}\right)} u_{n} y^{\mathcal{S}(\alpha)}
$$

Conversely, for any substitution $\theta=\left(u^{i}, u^{j}\right)$ and numbers $\mathcal{D}, \mathcal{P}$ and $\mathcal{S}$ satisfying the conditions $(*)$ and $(* *)$, it is easy to check that the derived substitution $\varphi$ generates $\xi=w^{\infty}$, where $w=u_{1} y^{\mathcal{D}\left(u_{1}, u_{2}\right)} \cdots y^{\mathcal{D}\left(u_{n-1}, u_{n}\right)} u_{n} y^{\mathcal{C}}$ with $u=u_{1} \cdots u_{n}$ and $\mathcal{C}$ the constant in (**). This is just the case (ii) in Theorem 2.7.

Subcase II.2. $\quad \theta(a)=(a x)^{i} a, \theta(x)=(x a)^{j} x$ with $i, j \geq 1$. 
Just as in Subcase II.1, $y \not \Varangle \varphi(a), y \not \varphi(a), y \not \varphi \varphi(x), y \not \varphi(x)$. And we have

- $\varphi(a)=\left(a y^{m} x y^{n}\right)^{i} a, \varphi(x)=\left(x y^{n} a y^{m}\right)^{j} x, \varphi(y)=y(m, n \geq 0, i, j \geq 1)$, and $\xi=\left(a y^{m} x y^{n}\right)^{\infty}$.

This is the case (i) in Theorem 2.7.

Subcase II.3. $\quad \theta(a)=\left(a x^{i}\right)^{j} a, \theta(x)=x$ with $i, j \geq 1$.

Consider $\varphi(x)$. Just as before, $y \not \varphi(x), y \not \varphi(x)$. Then $\varphi(x)=x$ and the letter $x$ is bounded. This is a contradiction.

Case III. Two letters are bounded.

Theorem 2.8. Let $\varphi$ be a non-ultimately cyclic substitution over $S$. Suppose that $\varphi$ generates, with the axiom a, a periodic sequence $\xi$, and $\xi$ contains any letter for infinite times. If two letters are bounded for $\varphi$, then there is a permutation $(x, y)$ of the letters $(b, c)$ such that $\varphi$ has one of the following forms:

(i) $\varphi(a)=(a u)^{k} a, \varphi(x)=x, \varphi(y)=y\left(u \in\{x, y\}^{+}, k \geq 1\right)$, and $\xi=(a u)^{\infty}$;

(ii) $\varphi(a)=a u_{1} a \cdots a u_{k} a, \varphi(x)=x, \varphi(y)=x\left(u_{i} \in\{x, y\}^{+},\left|u_{1}\right|=\cdots=\left|u_{k}\right|=\right.$ $L, k \geq 1)$, and $\xi=\left(a u_{1} a \cdots a u_{k} a x^{L}\right)^{\infty}$.

Proof. In this case, the letters $b$ and $c$ are bounded. There are three subcases:

Subcase III.1. $\varphi(b)=b$ and $\varphi(c)=c$.

Write $\varphi(a)=a u_{1} a \cdots a u_{k} a u_{k+1}$, where $u_{i} \in\{b, c\}^{*}$.

Since $u_{k+1}^{n} \triangleright \varphi^{n}(a)$ for any $n$, we have $u_{k+1}=\varepsilon$.

Take $n$ large enough such that $\varphi^{n}(a)=w^{l} r$ with $r$ a proper prefix of $w$ and $l \geq 1$. And the formula

$$
\varphi^{n+1}(a)=\varphi^{n}(a) u_{1} \varphi^{n}(a) \cdots \varphi^{n}(a) u_{k} \varphi^{n}(a)
$$

implies that $w^{l} r u_{1} w^{l} r \cdots w^{l} r u_{k} w^{l} r \prec w^{\infty}$. Recalling that $w$ is a primitive word and $\operatorname{Alph}(w)=\{a, b, c\}$, and noticing that $u_{i} \in\{b, c\}^{+}$, we have $r u_{1}=\cdots=$ $r u_{k}=w$. Hence $u_{1}=\cdots=u_{k}$. So we have

$$
\text { - } \varphi(a)=(a u)^{k} a, \varphi(b)=b, \varphi(c)=c\left(u \in\{b, c\}^{+}, k \geq 1\right) \text {, and } \xi=(a u)^{\infty} \text {. }
$$

This is the case (i) in Theorem 2.8 .

Subcase III.2. $\varphi(b)=c$ and $\varphi(c)=b$.

In this case $\varphi^{2}$ fulfils the condition in Subcase III.1, then $\varphi^{2}(x)=(x u)^{k} x$ with $u \neq \varepsilon$. But it is easy to see that this is impossible.

Subcase III.3. $\varphi(b)=\varphi(c) \in S$.

Suppose that $\varphi(b)=\varphi(c)=x \in S$. Write that $\varphi(a)=a u_{1} a \cdots a u_{k} a u_{k+1}$, where $u_{i} \in\{b, c\}^{*}$.

Since $x^{n\left|u_{k+1}\right|} \triangleright \varphi^{n+1}(a)$, we have $u_{k+1}=\varepsilon$.

Take $n$ large enough such that $w \triangleleft \varphi^{n}(a)$. And, as in Subcase III.1, the formula

$$
\varphi^{n+1}(a)=\varphi^{n}(a) x^{\left|u_{1}\right|} \varphi^{n}(a) \cdots \varphi^{n}(a) x^{\left|u_{k}\right|} \varphi^{n}(a)
$$

implies that $\left|u_{1}\right|=\cdots=\left|u_{k}\right|$. 
So we have

- $\varphi(a)=a u_{1} a \cdots a u_{k} a, \varphi(x)=x, \varphi(y)=x\left(u_{i} \in\{x, y\}^{+},\left|u_{1}\right|=\cdots=\right.$ $\left.\left|u_{k}\right|=L, k \geq 1\right)$, and $\xi=\left(a u_{1} a \cdots a u_{k} a x^{L}\right)^{\infty}$.

This is the case (ii) in Theorem 2.8 .

Now combining Theorems 2.1, 2.3, 2.5, 2.7 and 2.8, we get the proof of our main Theorem 0.2 .

\section{Remarks}

In this section, we give some remarks concerning the periodicity of a fixed point of a (possibly erasing) substitution.

\section{- Fixed point of a substitution}

Let $\varphi$ be a non-erasing substitution over $S$. If $\varphi$ generates a sequence $\xi$, say $\xi=\varphi^{\infty}(a)$, then $\varphi(\xi)=\xi$. In general, we say that a substitution $\varphi$ fixes an infinite sequence $\xi$ or that $\xi$ is a fixed point of $\varphi$ if $\varphi(\xi)=\xi$.

Obviously, a fixed point of $\varphi$ is also a fixed point of $\varphi^{n}$ for any $n \geq 1$.

Let $\varphi$ be a non-erasing substitution, and $\xi$ be a fixed point of $\varphi$. Write

$$
\xi=\xi_{1} \xi_{2} \xi_{3} \ldots \quad\left(\xi_{i} \in S\right)
$$

There are two cases:

Case I. The letter $\xi_{i}$ is bounded for any $i \geq 1$.

In this case, taking the formula $\varphi(\xi)=\xi$ into account, a simple induction on $i$ implies that $\varphi\left(\xi_{i}\right)=\xi_{i}$ for any $i$.

Case II. There is some letter $\xi_{i}$ which is growing.

Put $n=\min \left\{i \geq 1: \xi_{i}\right.$ is growing $\}$. Then we have $\varphi\left(\xi_{i}\right)=\xi_{i}$ for $i<n$, and the substitution $\varphi$ generates, with the axiom $\xi_{n}$, the sequence $\xi_{n} \xi_{n+1} \xi_{n+2} \cdots$

From the above characterization of the fixed point, we have

Theorem 3.1. Let $\varphi$ be a non-erasing substitution, and $\xi$ be a fixed point of $\varphi$. Then $\xi$ is ultimately periodic if and only if one of the following conditions holds:

1. $\varphi$ generates $\xi$, and $\xi$ is ultimately periodic;

2. $\xi=w \eta$, where $w \in S^{+}$with $\operatorname{Alph}(w) \varsubsetneqq S ; \eta \in S^{\mathbb{N}}$, and $\left.\varphi\right|_{\operatorname{Alph}(w)}$ is the identity; $\varphi$ generates the ultimately periodic sequence $\eta$;

3. there is a nonempty subset $X$ of $S$ such that $\left.\varphi\right|_{X}$ is the identity, and $\xi$ is any ultimately periodic sequence over $X$.

\section{- Erasing substitution}

We consider the erasing substitutions over $S=\{a, b, c\}$. There are several cases depending on the number of empty words among $\varphi(a), \varphi(b)$ and $\varphi(c)$ :

Case I. $\varphi(a)=\varphi(b)=\varphi(c)=\varepsilon$.

In this case, the substitution $\varphi$ fixes nothing but $\varepsilon$. 
Case II. Two of $\varphi(a), \varphi(b)$ and $\varphi(c)$ are empty, say $\varphi(b)=\varphi(c)=\varepsilon$.

In this case, if $a \notin \operatorname{Alph}(\varphi(a))$, then $\varphi^{2}$ fulfils the condition in Case I, and thus $\varphi$ fixes nothing but $\varepsilon$.

If $a \in \operatorname{Alph}(\varphi(a))$, then the periodic sequence $(\varphi(a))^{\infty}$ is the unique fixed point of $\varphi$.

Case III. Just one of $\varphi(a), \varphi(b)$ and $\varphi(c)$ is empty, say $\varphi(c)=\varepsilon$.

Define a morphism $\pi:\{a, b, c\}^{*} \rightarrow\{a, b\}^{*}$ as $\pi(a)=a, \pi(b)=b$, and $\pi(c)=\varepsilon$. Then $\theta=\pi \circ \varphi$ is a substitution over $\{a, b\}$.

Suppose that $\xi$ is a fixed point of $\varphi$, then $\varphi$ contains at least one letter in $\{a, b\}$ for infinitely many times (otherwise $\varphi(\xi)$ will be a finite word). Thus we can write

$$
\xi=z^{l_{1}} u_{1} z^{l_{2}} u_{2} \cdots \quad\left(l_{i} \geq 0, u_{i} \in\{a, b\}\right) .
$$

Therefore $\xi=\varphi(\xi)=\varphi\left(u_{1} u_{2} \cdots\right)$, and thus

$$
u_{1} u_{2} \cdots=\pi(\xi)=\pi\left(\varphi\left(u_{1} u_{2} \cdots\right)\right)=\theta\left(u_{1} u_{2} \cdots\right) .
$$

Without loss of generality, we assume that $u_{1}=a$, and thus $a \triangleleft \theta(a)$.

Subcase III.1. $\theta(b)=\varepsilon$, or $\varphi(b) \in c^{+}$.

In this subcase $\varphi^{2}$ fulfils the condition in Case II, and it is easy to see that $\left(\varphi^{2}(a)\right)^{\infty}$ is the unique fixed point of $\varphi$.

Subcase III.2. $\theta$ is non-erasing over $\{a, b\}$.

In this subcase, the sequence $\eta=u_{1} u_{2} \cdots$ is a fixed point of the non-erasing substitution $\theta$.

Since $\eta=\pi(\xi)$ and $\xi=\varphi(\eta)$, the sequence $\xi$ is ultimately periodic if and only if $\eta$ is ultimately periodic.

Up to now, we have shown that

Theorem 3.2. Let $\varphi$ be an erasing substitution over $S=\{a, b, c\}$, and $\xi$ be $a$ fixed point of $\varphi$. Then $\xi$ is ultimately periodic if and only if there is a permutation $(x, y, z)$ of the letters $(a, b, c)$ such that one of the following conditions holds:

1. $\varphi(x) \in S^{+}, \varphi(y)=\varphi(z)=\varepsilon$ with $|\varphi(x)|_{x} \geq 1$, and $\xi=(\varphi(x))^{\infty}$;

2. $\varphi(x) \in S^{+}, \varphi(y) \in z^{+}, \varphi(z)=\varepsilon$ with $z^{l} x \triangleleft \varphi(x)$ for some $l \geq 0$, and $\xi=\left(\varphi^{2}(x)\right)^{\infty}$;

3. $\varphi(x)=u, \varphi(y)=v, \varphi(z)=\varepsilon$ with the words $u, v \in S^{+}$such that the substitution $\theta$ over $\{x, y\}$ defined by $\theta(a)=\pi(u)$ and $\theta(b)=\pi(v)$ is non-erasing and fixes a ultimately periodic sequence $\eta \in\{x, y\}^{\mathbb{N}}$, and $\xi=\varphi(\eta)$.

Where the morphism $\pi$ is defined as $\pi(x)=x, \pi(y)=y$ and $\pi(z)=\varepsilon$.

\section{- Substitution fixing a finite word}

Let $\varphi$ be a substitution. If there is a word $w \in S^{+}$such that $\varphi(w)=w$, we say that $\varphi$ fixes a finite word. And in this case, we have that $\varphi$ fixes the periodic sequence $w^{\infty}$. The interested reader is referred to [3] for a characterization of such substitutions. 
Acknowledgements. We would like to thank the anonymous referees of the present paper for their valuable remarks which have improved the readability of the paper.

\section{REFERENCES}

[1] N.J. Fine and H.S. Wilf, Uniqueness theorem for periodic functions. Proc. Amer. Math. Soc. 16 (1965) 109-114.

[2] T. Harju and M. Linna, On the periodicity of morphisms on free monoids. RAIRO-Theor. Inf. Appl. 20 (1986) 47-54.

[3] T. Head, Fixed languages and the adult language of OL schemes. Int. J. Comput. Math. 10 (1981) 103-107.

[4] B. Lando, Periodicity and ultimate periodicity of D0L systems. Theor. Comput. Sci. 82 (1991) 19-33.

[5] M. Lothaire, Combinatorics on Words. Encyclopedia of Mathematics and its Applications, Vol. 17, Addison-Wesley (1983).

[6] J. Pansiot, Decidability of periodicity for infinite words. RAIRO-Theor. Inf. Appl. 20 (1986) 43-46.

[7] G. Rozenberg and A. Salomaa, The Mathematical Theory of L Systems. Academic Press, New York (1980).

[8] P. Séébold, An effective solution to the D0L periodicity problem in the binary case. EATCS Bull. 36 (1988) 137-151.

Received January 18, 2007. Accepted November 23, 2007. 